\title{
Evaluation of functional outcome of patients with fracture neck of femur treated with Austin Moore prosthesis at government medical college, Thrissur-a prospective study during October 2019 to October 2020
}

\author{
Jyothish Kavungal*, Nihal Suresh \\ Department of Orthopaedics, Government Medical college, Thrissur, Kerala, India \\ Received: 22 October 2021 \\ Revised: 23 December 2021 \\ Accepted: 01 February 2022

\section{*Correspondence:} \\ Dr. Jyothish Kavungal, \\ E-mail: jyothishkavungal@gmail.com \\ Copyright: (C) the author(s), publisher and licensee Medip Academy. This is an open-access article distributed under \\ the terms of the Creative Commons Attribution Non-Commercial License, which permits unrestricted non-commercial \\ use, distribution, and reproduction in any medium, provided the original work is properly cited.
}

\begin{abstract}
Background: Femoral neck fractures commonly affects the elderly population. $90 \%$ of femoral neck fractures in the elderly result from simple fall. Elderly age group suffers from innumerable illnesses such as diabetes, hypertension, coronary artery disease, chronic obstructive pulmonary disease, all of which can aggravate following a femoral neck fracture because patient can become completely bedridden. The aim of treating these patients is to make them mobilize as early as possible to achieve his or her pre-fracture functional status. The incidence of femoral neck fractures is increasing dramatically as the mean age of the population increases. Prosthetic replacement allows immediate weight bearing to elderly patients and thus helps to avoid complications of recumbency and inactivity. To study the functional outcome in patients with femoral neck fractures treated with Hemiarthroplasty using Austin Moore prosthesis.

Methods: Prospective study was done among 42 consented patients who sustained neck of femur fractures and were admitted in department of orthopaedics, government medical college, Thrissur, between 01/10/19 to 01/10/20, who were aged above 60 years and presenting within 3 weeks of injury. Patients were followed up at an interval of 6 weeks, 3 months and 6 months functional outcome was analysed by modified Harris hip scoring system.

Results: Among the 42 subjects, $52.4 \%$ were females and the rest were males. Mean age of the study population was $71.57 \pm 7.047$ years. Harris hip score at end of 6 months ranged from 35 to $94.33 .3 \%$ of hemiarthroplasties had hip score of $91-100$ (excellent), $38.1 \%$ of the hemiarthroplasties had scores of $81-90$ (good), $19 \%$ had satisfactory/fair results whereas only $9.5 \%$ of hemiarthroplasties fell in poor category with scores below 70 .

Conclusions: Hemiarthroplasty with Austin Moore prosthesis is still a good option in elderly patients with femoral neck fractures.
\end{abstract}

Keywords: Hemiarthroplasty, Harris hip score

\section{INTRODUCTION}

Hip fractures are devastating injuries that mostly affect the elderly and have a tremendous impact on both the health care system and society in general. Approximately $50 \%$ of all hip fractures are intracapsular fractures of femoral neck. ${ }^{1}$ The incidence of femoral neck fractures is increasing dramatically as the mean age of the population increases. It has been predicted that by 2050 , the number of hip fractures would triple its present size. As a consequence, proximal femur fractures are a significant cause of mortality and morbidity in all age groups, especially in elderly. ${ }^{2}$

Ideal treatment of intracapsular fractures of proximal femur is anatomical reduction and stable internal fixation. 
Hip replacement arthroplasty (partial or total) is emerging as the most viable treatment option for the elderly. ${ }^{3}$ Prosthetic replacement allows immediate weight bearing to elderly patients and thus helps to avoid complications of recumbency and inactivity.

Hemiarthroplasty can be used for both femoral neck and basi-cervical neck fractures. It is used as a salvage operation for non-healed femoral neck fractures in elderly patients. The hemiprosthesis can be of a unipolar or a bipolar design. In unipolar prosthesis, a hemi head is attached directly to the stem through a taper locking mechanism, permitting movement only in the hip joint.

A bipolar hemiprosthesis consists of a femoral stem, a femoral head and a bipolar head. The femoral head can be in one piece together with the stem, or it can be attached to the stem through a taper locking mechanism. The latter giving the advantage of adjusting tension by choosing between different sizes of the head.

Various designs of prosthetic replacement have been devised. The Moore (AMP) type being the unipolar endoprosthesis introduced in 1950. Bateman in 1974 reported the bipolar or universal endoprosthesis.

Operative management consisting of fracture reduction and stabilisation, which permits early patient mobilisation and minimises many of the complications of prolonged bed rest, has become the treatment of choice for most of the femoral neck fractures. There nevertheless remain situations in which surgery cannot be performed and treatment must be non-operative.

Replacement arthroplasty is a very useful procedure and has to be carried out when it is not possible to achieve union of fracture or in old people who has to be put on their feet without loss of time. Prosthetic replacement allows immediate weight bearing to elderly patients and thus helps to avoid complications of recumbency and inactivity.

This study tried to evaluate the functional outcome of patient treated for hip fractures using the Austin Moore prosthesis.

\section{Aims and objectives}

Aim and objectives were to study the functional outcome of neck of femur fractures treated with hemiarthroplasty using Austin Moore prosthesis in patients above 60 years of age, attending the orthopaedics department in government medical college, Thrissur from 01/10/19 to $01 / 10 / 20$ and to study how early mobilization of the patients can be done.

\section{METHODS}

This prospective observational study was done at department of orthopaedics, government medical college, Thrissur, Kerala during 01/10/2019 to 01/10/2020. Study population include patients attending department of orthopaedics, government medical college, Thrissur, fulfilling the inclusion criteria during the study period.

Sample size was calculated based on the formula $4 \mathrm{pq} / \mathrm{d}^{2}$ with $95 \%$ confidence interval and 5 percentage alpha error. ' $\mathrm{P}$ ' was taken as proportion of patients with good to excellent results in study by Arwade et al which was given as $70 \% .{ }^{19}$ Maximum allowable error (d) was taken as $20 \%$, and the subsequent sample size calculated was 42 .

\section{Inclusion criteria}

Consenting patient with fracture neck of femur attending orthopaedics department of government medical college, Thrissur.

Patient age above 60 years, patient presenting within 3 weeks of injury were included in the study.

\section{Exclusion criteria}

Patients with associated neurovascular injury, patients with associated fractures in the same limb, patients with history of previous surgery in the fractured area and patients with pre-existing musculoskeletal disease were excluded from the study.

The patient data are obtained according to the proforma, which includes age, sex, socioeconomic status, Occupation, nature and side of the fracture, type of fracture and associated injuries and comorbidities. After proper history taking, clinical examination, radiological work up, preoperative work up and pre-anaesthetic check-up was done in all patients. Patients were followed up at an interval of 6 weeks, 3 months and 6 months functional outcome was analysed by modified Harris hip scoring system. Serial radiographs were taken for each visit.

All data obtained during the study was entered into MS excel (version 2007) after coding. Statistical analysis was done after exporting the coded data into Epi Info by C.D.C. Categorical variables were summarized in terms of frequencies and percentages, while continuous data are presented as the mean with the standard deviation.

\section{Ethical concerns and conflict of interests}

Clearance from IEC/IRB was obtained Informed written consent was taken from the subjects and the guardians/relatives of all the patients in this study. As a result of taking part in this study the patient has not been put under any increased risk.

No conflict of interest in the current study.

\section{Preoperative management}

Patients usually present in casualty and were admitted to the ward. Detailed history was taken with particular 
emphasize on mode of injury and associated medical illness. Detailed clinical assessment was done in each case.

In all patients, preoperatively skin traction with $2.5 \mathrm{~kg}$ weight was applied, to the fractured lower limb, with the aim of relieving pain and preventing shortening and to reduce unnecessary movements of the injured limb. Oral or parental NSAIDs were given to relieve the pain.

Antero-posterior radiographs of the affected hip joint of pelvis with both hips were taken for all the patients.

Routine investigations like complete blood count, blood grouping, renal function test, liver function test, urine routine, random blood sugar, serum electrolytes, screening for HBSAg, HIV and HCV, chest x-ray and ECG were done. Appropriate consultation and treatment were done according to co morbidities such as diabetes, hypertension, coronary heart disease, chronic obstructive pulmonary disease, chronic liver disease, asthma, renal failure etc. These patients are well evaluated before surgery.

Certain therapeutic exercises were taught preoperatively to the patients which had to be continued postoperatively, such as deep breathing exercises, static quadriceps exercises and ankle movements.

The details and complications of surgery were explained to the patient and by stander for obtaining written informed consent. Before starting the surgery, a single dose of Intravenous antibiotics and tetanus immunization were given. Parts were prepared from umbilicus to knee joint.

\section{Surgical procedure}

All surgical procedure was performed on an elective basis with all asepticprecautions under spinal or general anaesthesia.

\section{Position of the patient}

Preferred position of patient was lateral position, lying on the unaffected side. The skin over the affected limb was scrubbed with betadine from knee to hip. The operating lower limb was draped with sterile cloth separately for easy manipulation.

\section{Moore's approach (Southern exposure)}

For all patients' posterior approach (Moore's approach also labelled as Southern exposure) was used in present series.

A curved skin incision of $10-15 \mathrm{~cm}$ beginning from $10 \mathrm{~cm}$ distal to posterior superior iliac spine directed to the posterior edge of the greater trochanter and continued down along the shaft of femur.

Deep fascia and the fascia over the gluteus maximus were divided along the skin incision, blunt dissection of gluteus maximus was done along its fibres.
The sciatic nerve was felt by finger palpation and retracted away which was not exposed.

The fibres of the gluteus maximus were retracted to both the sides and greater trochanter was exposed. The short external rotators (gemelli, obturator internus and the piriformis tendon) were tagged and cut near their insertion at the trochanter.

The exposed posterior capsule was opened by a $\mathrm{T}$ shaped incision. The head is delivered out using bone levers or corks screw after adducting and internally rotating femur.

Head size is measured using sizer and confirmed using a trial prosthesis by its suction fit in acetabulum. Femoral shaft is prepared using rasp.

After preparing acetabulum by cutting ligamentum teres and clearing soft tissue, implant is inserted by maintaining femoral anteversion.

Hip is reduced by traction of thigh and assessed for range of motion and stability. The capsule was sutured then.

The tagged short external rotators were re sutured by bone tunnels in the remaining trochanter.

Absolute haemostasis was obtained.

\section{Postoperative management}

The legs were kept abducted using a pillow in between. Regular half hourly, Temperature, pulse rate, respiratory rate and blood pressure charts were maintained, 4th hourly for initial 24 hours.

Antibiotics in the form of parenteral cefaperazone sulbactam $1.5 \mathrm{gm}$, two times a day was given for the first three days and later shifted to oral antibiotics. On the first postoperative day, intramuscular analgesics were given. Deep breathing exercise was advised. Drain removal was done after 48-72 hours depending upon collection.

A postoperative check X-ray was taken. Knee flexion, isometric quadriceps exercises and hip abduction, flexion and extension exercises were started on $2^{\text {nd }}$ postoperative day. The patients were allowed to sit on the second day, and on the third day they were mobilized with partial weight bearing with the help of a walker.

The patient was advised to use a straight high chair with arms to facilitate getting out of the chair and avoid using a sofa. The patient was advised not to sit cross legged or squat on the floor or squat for Indian style of toilet and patient was advised to use elevated toilet seat. Suture removal was done on the tenth postoperative day and the patient was discharged from the hospital.

The patient who had infection were treated accordingly before discharging them from the hospital. 


\section{Follow up}

Patients were followed up at an interval of 6 weeks, 3 months and 6 months functional outcome was analysed by modified Harris hip scoring system. Serial radiographs were taken for each visit.

\section{RESULTS}

A total of 42 subjects who underwent surgery for fracture neck of femur were included in the study. The study population had participant with maximum age of 84 years old. The mean age of the study population was $71.57 \pm$ 7.047 years. The numbers of females were 22 and males were 20 in the present series, which shows that $52.4 \%$ were females in this study. The female preponderance is higher compared to the males.

Left side was more affected than right side. There were 23 left sided fractures and 19 right sided fractures. Out of all the study subjects 37 of the patients sustained the fracture following a history of trivial trauma and only 5 of them sustained it due to a road traffic accident (RTA).

The study found that 15 patients in the study population had co-morbidities. The common ones include diabetes, hypertension, respiratory diseases like COPD and cardiovascular diseases like ischemic heart disease (IHD). Among comorbidities $28.6 \% \quad(n=12)$ had history of hypertension followed by diabetes which accounted for $16.7 \%(n=7)$ of the study population. These patients were first worked up and had undergone consultations from the associated departments. They were stabilised and fitness for anaesthesia and surgery, from concerned departments, were obtained before being taken up for surgery.

Depending upon the radiological examination, study found that $67 \%(n=28)$ as type 3 and $33 \%(n=14)$ were of type 4 femoral neck fractures. Fractures were classified according to Gardens classification.

Few complications were noted during follow up. The $5 \%$ $(n=2)$ patients developed bed Sores, who were treated according to culture and sensitivity, appropriate antibiotics and sterile dressing. The $5 \%(n=2)$ patients had superficial infections, none of them had deep seated infections. The $2 \%(n=1)$ patient had posterior dislocation which was reduced under GA. The $88 \%(n=37)$ of patients didn't have any complications.

Table 1: Distribution of sample by criteria of complications.

\begin{tabular}{|lll|}
\hline Complications & Frequency & Percentage (\%) \\
\hline Bed sore & 2 & 4.8 \\
\hline $\begin{array}{l}\text { Posterior } \\
\text { dislocation }\end{array}$ & 1 & 2.4 \\
\hline $\begin{array}{l}\text { Superficial } \\
\text { infection }\end{array}$ & 2 & 4.8 \\
\hline Total & 42 & 100.0 \\
\hline
\end{tabular}

The $69 \%$ of the patients had a hospital stay of less than 20 days. Patients were discharged only after assessment of pain, limb length discrepancy, mobilization and radiological assessment. Patients were mobilized with a walker on post op day 3 and then with or without a walker or crutch following the functional outcome of the hemiarthroplasty.

The functional outcome after hemiarthroplasty using AMP prosthesis for intracapsular fracture neck of femur was graded as excellent, good, fair and poor after adding the scores given for each criterion of assessment of hip, using modified Harris Hip score. In the present study, Harris hip score at end of 6 months ranged from 35 to $94.33 .3 \%$ of hemiarthroplasties had hip score of 91-100 (excellent), $38.1 \%$ of the hemiarthroplasties had scores of 81-90 (good), 19\% had satisfactory/fair results whereas only $9.5 \%$ of hemiarthroplasties fell in poor category with scores below 70 .

Table 2: Distribution of sample by criteria of functional results.

\begin{tabular}{|lll|}
\hline Harris hip score & Frequency & Percentage (\%) \\
\hline Excellent & 14 & 33.3 \\
\hline Good & 16 & 38.1 \\
\hline Fair & 8 & 19.0 \\
\hline Poor & 4 & 9.5 \\
\hline Total & 42 & 100.0 \\
\hline
\end{tabular}

It was found that $71.4 \%$ of the study subjects $(n=30)$ had either excellent or good outcome post operatively as per the Harris hip score.
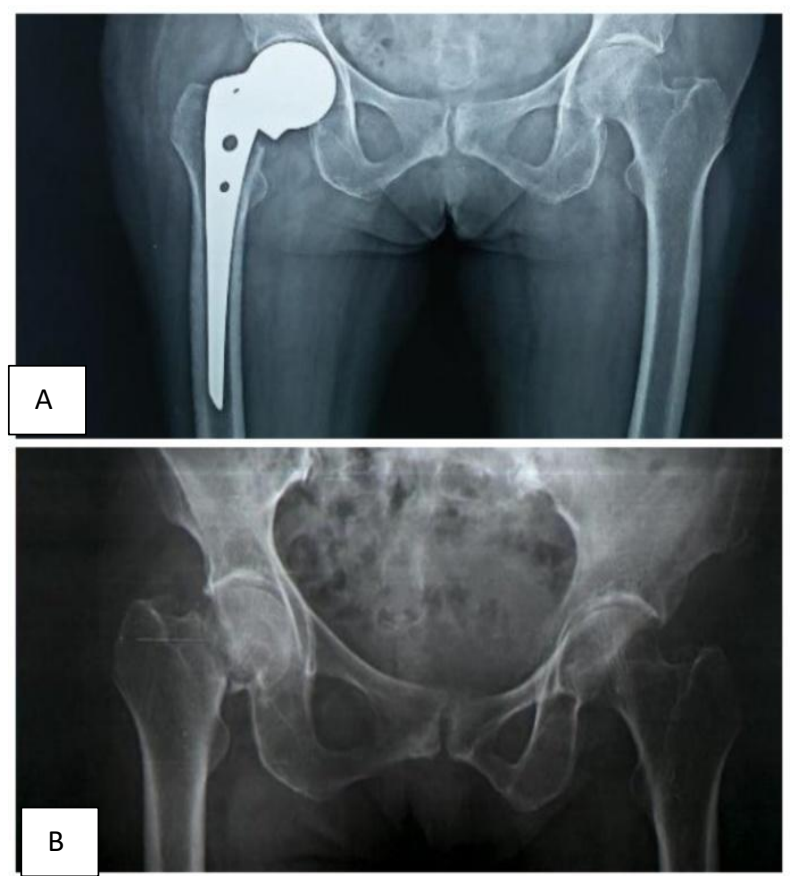

Figure 1 (A and B): Post operative and pre-operative radiographs showing fracture neck of femur treated with Austin Moore prosthesis. 


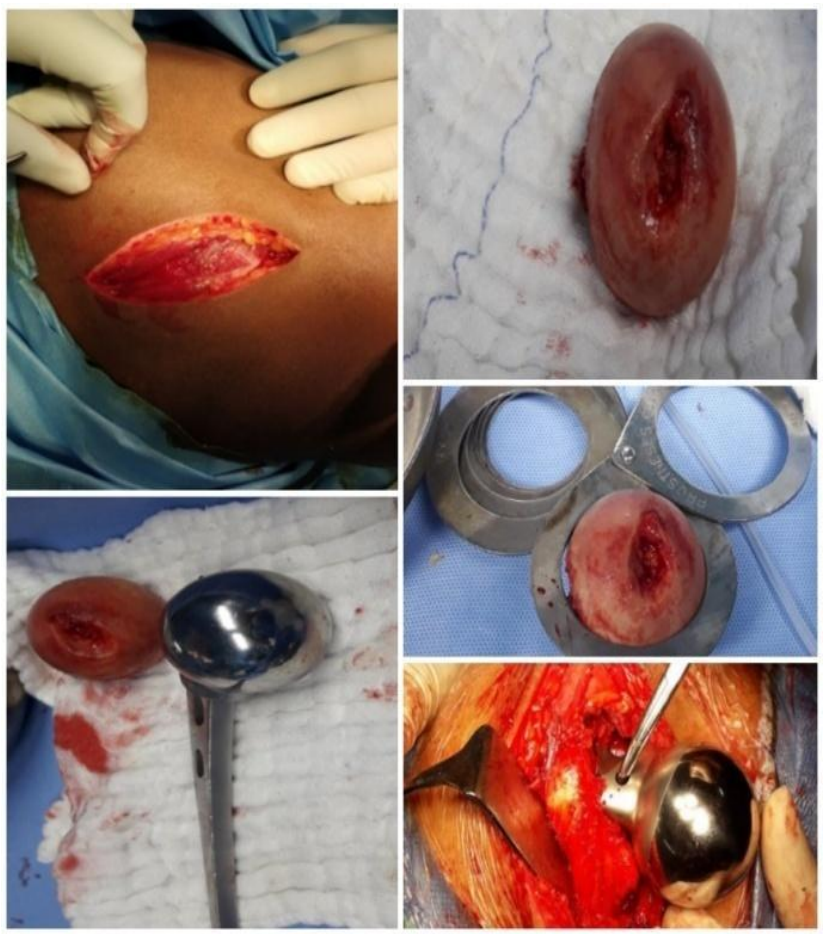

Figure 2: Intra operative images.

The Figure 2 shows lateral position and Moore's approach incision, delivery of femoral head and delivery of femoral head in sizer, selection and insertion of Austin Moore prosthesis in clockwise direction.
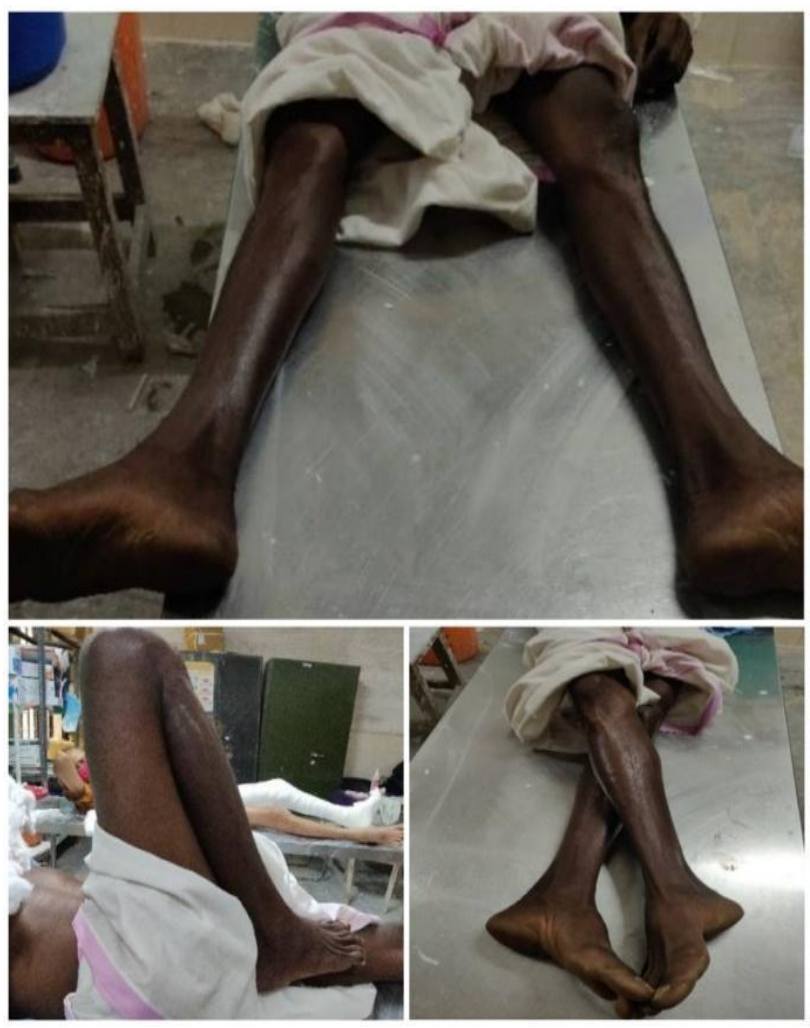

Figure 3: Range of movements in operated hip during $6^{\text {th }}$ month follow up.

\section{DISCUSSION}

Femoral neck fractures are one of the most common fractures seen in elderly age group. They are one of the major causes of morbidity and mortality. Fracture neck of femur is still an unsolved enigma for an orthopaedic surgeon. Results have been variable with various modalities of treatment which includes osteosynthesis, hemiarthroplasty and total hip replacement.

It is dissatisfaction of many surgeons with the above methods of treatment particularly in older people, that lead to development of hip prosthesis as a final procedure in developing a painless, functional and stable hip. The rationale of this procedure is based on the observation that the hip functions fairly, following salvage procedure in which an endoprosthesis has been used for various pathological conditions.

The final goal in any replacement surgery is to facilitate an early return to activities of daily life during the pre-fracture levels. Since osteosynthesis is not a very good idea for elderly population, as secondary procedure may be required on its failure and elderly patients may not be able to tolerate effects of second surgery hemiarthroplasty continues to be a favoured option.

The present study is to evaluate the results of hemiarthroplasty using in fracture neck of the femur using Austin Moore prosthesis keeping in view of the living condition of an average Indian.

The functional outcome was assessed using Harris hip score.

\section{Modified Harris hip scoring system}

Total functional outcome was graded as following depending on the total: Pain relief, function, range of motion and absence of deformity.

With the above parameters, total points were calculated. Maximum points from the Harris hip score would be 100 .

Based on the scoring, functional outcomes were graded as follows: Excellent-Harris hip score between 1-100, goodHarris hip score between 81-90, fair-Harris hip score between 71-80 and poor-Harris hip score less than 70 .

The present study found that average age of patients as 72 years. Majority of the patients were between 70-79 years. Similar age distribution is reported by other authors. ${ }^{4-6}$ Saxena et al reported a mean age of 66 years in their study, while the mean age was 65 years in the study done by Mukherjee et al, Bavadekar et al in their study found the mean age as 75 years which is somewhat closer to the results obtained in the present study. ${ }^{4-6}$ In the present study, femoral neck fractures were found to be more in females $(52 \%)$. Female preponderance has been reported in several case series. ${ }^{7,8}$ Left sided hip was fractured in 23 
patients (55\%). Boyd et al reported $55 \%$ fractures on left side and D'Acry et al reported $55.4 \%$ fractures on left side. ${ }^{9,10}$ The common comorbid conditions noted in this study were diabetes, hypertension, IHD and COPD. 64\% of patients in this study had one or many of these problems. The $5 \%$ of patients had respiratory diseases like COPD, $17 \%$ of them had diabetes, $29 \%$ had hypertension, $5 \%$ had cardiac problems. The common conditions in this study were diabetes, hypertension, IHD, and COPD. Hinchey and Day reports hypertension prevalence as $13.9 \%$ and $13.26 \%$ diabetes mellitus in their study population. ${ }^{11}$

Majority of cases $88 \%$ were due to trivial trauma like fall, rest of the cases were due to severe trauma like RTA. This is in accordance with majority of the series reportedEvarts, Inghalikar et al, Seth, Stevens et al and several other authorities believe that the intracapsular fracture are stress fractures through pathological bone secondary to osteoporosis or osteomalacia. ${ }^{12,13,20,21}$

In this study hospital stay ranges from 11 to 28 days with a mean average of 18 days. Stinchfield et al reported mean average of 31.5 days hospital stay, Klenerman et al reported 28 days, Savastano et al reported 38 days. ${ }^{14-16}$ The mean duration of hospital stay was similar to the studies done in different parts of the country.

Complications after hemiarthroplasty may be divided into two categories broadly 1) Early complications and 2) Late complications. Early complications like nerve and vascular injuries, hematoma formation and haemorrhage, bladder injury, limb length discrepancy, infections, thromboembolism and dislocations. Late complications such as hetero-tropic-ossification, implant loosening, acetabular protrusion, erosion etc may occur rarely. 88.1\% of the study subjects in the present study didn't have any complications which might be due to proper case selection and prompt post-operative care and follow up.

There were no post-operative deaths in the present study. Wai Hee et al had $4 \%$ mortality due to sepsis. ${ }^{17}$ Salvatti et al had high mortality following infection of prosthesis. ${ }^{18}$ No mortality in this study maybe due to proper selection of cases, proper management of associated medical problems preoperatively, use of antibiotics routinely, and early mobilisation. There were only 2 cases of superficial infections which was managed with sterile dressings and appropriate antibiotics.

The functional outcome in this study was assessed at the end of 6 months using modified Harris hip score. According to this, $33.3 \%$ of study population had "excellent" functional outcome, 38.1\% had "good"; $19 \%$ had "fair" and 9.5\% had "poor" functional outcome. Similar results were reported in a study by Arwade et al. ${ }^{19}$ Hinchey and Day reported excellent functional outcome among $52.4 \%$ of their study population. ${ }^{11}$ Salvatti et al reported that $31 \%$ reported excellent functional outcome, Saxena and Saraf reported that $46.1 \%$ had excellent functional outcome. ${ }^{4}$
The difference between excellent and good results are minimal, so similar to the other studies conducted worldwide present study also tried to evaluate results by grouping excellent and good Harris hip scores. Hinchey in their study found that $72.6 \%$ had either good or excellent result in Harris hip score after grouping it, while Salvatti et al found this value to be as $57 \%$ in their study. ${ }^{11,18}$ Similarly, Saxena et al found $90.9 \%$ had good or excellent outcome after grouping them, Arwade et al 70\%, Mukherjee and Puri 78\%., 4,5 The present study found $71.4 \%$ had good or excellent result after grouping, which is comparable with other studies as mentioned above.

\section{CONCLUSION}

Forty-two cases of fracture neck of femur in elderly patients above the age of 60 years treated by hemiarthroplasty using AMP prosthesis in the department of orthopaedics at government medical college, Thrissur between $01 / 10 / 19$ to $01 / 10 / 20$. The cases were followed up for 6 months and the functional results were analysed by using modified Harris hip scoring system.

The success of hemiarthroplasty depends on preoperative planning and proper attention to surgical details to achieve the optimum biomechanical stability. The mortality and morbidity are not high, operative procedure is simple, complications are disabling and second operation is less frequently required. Thus, it can be concluded that hemiarthroplasty with Austin Moore prosthesis is still a good option in elderly patients with femoral neck fractures. Also, most of the population here comes from rural areas and belong to low socioeconomic class. So, such hemiarthroplasty with Austin Moore prosthesis provides a cost-effective method of surgical option for these patients.

\section{Funding: No funding sources \\ Conflict of interest: None declared}

Ethical approval: The study was approved by the institutional ethics committee

\section{REFERENCES}

1. Blomfeldt R, Torkvist H, Ponzer S, Soderquist A, Tidermark J. Internal Fixation versus hemiarthroplasty for displaced fractures of the femoral neck in elderly patients with severe cognitive impairment. J Bone Joint surg Br. 2005;87(4):523-9.

2. Harthesis JW. Arthroplasty of hip, chapter 7 in: Campbells operative orthopaedics $11^{\text {th }}$ edition. Mosby. 2008;312-481.

3. Sandhu HS. Management of Fracture neck of femur. Indian J orthop. 2005;39:130-6.

4. Saxena PS, Saraf JK. Moore Prosthesis in fracture neck of femur. Indian J Orthop. 1978;12:138-45.

5. Mukherjee DL, Puri HC. Early hemiarthroplasty for fresh fractures of the neck of the femur in geriatric patients. Indian J Surg. 1986;48:77-80. 
6. Bavadekar AV, Manelkar KR. Hemiarthroplasty of the hip in the treatment of intracapsular fracture neck of the femur state of the art and an appraisal. Clin Orthop India. 1987;1:43-52.

7. Moore AT. The self-locking metallic hip prosthesis. JBJS. 1957;39A:811-27.

8. Sikorski JM, Barrington. Internal fixation versus hemiarthroplasty for the displaced sub-capital fracture of femur: A prospective randomized study. J Bone Joint Surg Am. 1981;63B:357-61.

9. Boyd HB, Salvatore JE. Acute fractures of the femoral neck: Internal fixation or Prosthesis. J Bone Joint Surg Am. 1964;46A:1066-8.

10. D'Acry J, Devas M. Treatment of fractures of the femoral neck by replacement with the Thompson prosthesis. J Bone Joint Surg Am. 1976;58B:279-86.

11. Hinchey Day. Primary prosthetic replacement in fresh femoral neck Fractures. J Bone Joint Surg Am. 1960;42B:633-40.

12. Evarts CM. Endoprosthesis as the primary treatment of femoral neck fractures. Clinc Orthop. 1973;92:6976.

13. Seth MK. Stress fractures of the neck of femur. Clin Orthrop India. 1987;1:105-9.

14. Stinchfield FE, Cooperman B, Shea CE. Replacement of the femoral head by Judet or Austin Moore Prosthesis. JBJS 1957;39A:1043-58.
15. Klenerman L, Marcuson RW. Intracapsular fractures of the neck of the femur. J Bone Joint Surg Am. 1970;52B:514-7.

16. Savastano AA, Sage LA, Zecchino V. Treatment of fractures of the neck femur with intramedullary stem prosthesis. Arch Surg. 1957;75(6):156-9.

17. Wai-Hee Lo, Chen WM, Huang CK, Chen TH, Chiu FY, Chen CM. Bateman bipolar hemiarthroplasty for displaced intracapsular femoral neck fractures. Clin Orthop. 1994;302:75-82.

18. Salvatti EA, Artz T, Algeitti P, Asins SE. Endoprosthesis in the treatment of femoral neck fractures. Orthop. Clinc N Am. 1974;5:757-7.

19. Arwade DJ. A review of internal fixation and prosthetic replacement for fresh fractures of the femoral neck. Clini Orthop India. 1987;1:77-82.

20. Ingalhalikar VT, Kumta S. Fracture neck femur anatomical and biomechanical aspects. Clinc Orthop India. 1987.

21. Stevens J, Freeman PA, Nordin BEC, Barnett E. The incidence of osteoporosis in patients with femoral neck fractures J Bone Joint Surg Am. 1962;44-B:5207.

Cite this article as: Kavungal J, Suresh N. Evaluation of functional outcome of patients with fracture neck of femur treated with Austin Moore prosthesis at government medical college, Thrissur-a prospective study during October 2019 to October 2020. Int J Res Orthop 2022;8:151-7. 